\title{
Working memory training efficacy in COPD: the randomised, double-blind, placebo-controlled Cogtrain trial
}

\author{
Martijn van Beers $\mathbb{B}^{1}$, Sarah W. Mount ${ }^{1}$, Katrijn Houben ${ }^{2}$, Harry R. Gosker $\mathbb{1}^{1}$, Lisanne Schuurman ${ }^{1}$, \\ Frits M.E. Franssen $\mathbb{1}^{1,3}$, Daisy J.A. Janssen $\mathbb{1}^{3,4}$ and Annemie M.W.J. Schols ${ }^{1}$
}

${ }^{1}$ Dept of Respiratory Medicine, NUTRIM School of Nutrition and Translational Research in Metabolism, Maastricht University Medical Centre+, Maastricht, The Netherlands. ${ }^{2}$ Dept of Clinical Psychological Science, Faculty of Psychology and Neuroscience, Maastricht University, Maastricht, The Netherlands. ${ }^{3}$ Dept of Research and Education, CIRO, Horn, The Netherlands. ${ }^{4}$ Dept of Health Services Research, Care and Public Health Research Institute, Faculty of Health, Medicine and Life Sciences, Maastricht University, Maastricht, The Netherlands.

Corresponding author: Annemie M.W.J. Schols (a.schols@maastrichtuniversity.nl)

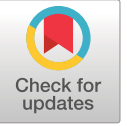

This version is distributed under the terms of the Creative Commons Attribution NonCommercial Licence 4.0. For commercial reproduction rights and permissions contact permissions@ersnet.org

This article has supplementary material available from openres.ersjournals.com

Received: 22 July 2021 Accepted: 13 Sept 2021

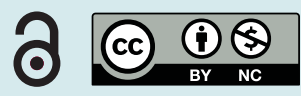

Shareable abstract (@ERSpublications)

Working memory training improves performance on the trained tasks but not overall cognitive performance, healthy lifestyle behaviours or cognitive stress susceptibility in patients with COPD https://bit.ly/3Erjlx2

Cite this article as: van Beers M, Mount SW, Houben $\mathrm{K}$, et al. Working memory training efficacy in COPD: the randomised, double-blind, placebo-controlled Cogtrain trial. ERJ Open Res 2021; 7: 004752021 [DOI: 10.1183/23120541.00475-2021].

\section{Abstract}

Background Cognitive impairment is highly prevalent in COPD and is associated with a sedentary lifestyle, unhealthy diet and increased cognitive stress susceptibility. Enhancement of cognitive performance by working memory training (WMT) may reverse these effects. Therefore, this study aimed to investigate the efficacy of WMT in COPD on cognitive performance, healthy lifestyle behaviours and cognitive stress susceptibility.

Methods The double-blind randomised, placebo-controlled Cogtrain trial consisted of a 12-week training phase comprising 30 active or sham WMT sessions, followed by a second 12-week maintenance phase with 12 sessions. Measurements took place at baseline and after the first and second phases. The primary outcome was cognitive performance. Secondary outcomes were the recall of prespecified healthy lifestyle goals, physical capacity and activity, dietary quality and cognitive stress susceptibility. Motivation towards exercising and healthy eating and psychological wellbeing were exploratory outcomes.

Results Sixty-four patients with moderate COPD (45\% male, aged 66.2 \pm 7.2 years, median forced expiratory volume in $1 \mathrm{~s} 60.6 \%$ predicted) were randomised. WMT significantly increased patients' performance on the trained tasks in the first phase, which remained stable in the second phase. Of the 17 cognitive outcome measures, only one measure of memory improved after the first phase and one measure of reaction time after the second phase. This intervention did not influence physical capacity and activity, recall of prespecified healthy lifestyle goals, psychological wellbeing or cognitive stress susceptibility.

Conclusion WMT improved performance on the trained tasks but not overall cognitive performance, healthy lifestyle behaviours or cognitive stress susceptibility in patients with COPD.

\section{Introduction}

COPD is characterised by persistent airflow limitation and chronic respiratory symptoms [1]. Muscular [2] and metabolic abnormalities [3, 4], anxiety, depression [5] and cognitive impairment are common comorbidities of COPD. A recent large review reported a 32\% prevalence of any cognitive impairment and a $25 \%$ prevalence of mild cognitive impairment in patients with COPD [6]. More recently, a $39.4 \%$ prevalence of cognitive impairment was reported in clinically stable patients [7] and a $41.5 \%$ [8] and $56.7 \%$ [9] prevalence in patients referred to pulmonary rehabilitation (PR). In contrast, the prevalence of cognitive impairment among non-COPD controls in the latter study was $13.3 \%$. Disease-specific and lifestyle factors including hypoxia, a history of smoking, dietary insufficiencies and sedentary behaviour may contribute to this elevated prevalence [10]. 
It is important to consider cognitive impairment in COPD management because it negatively impacts patients' health outcomes [11]. Lower working memory capacity, which refers to the ability to keep important information in mind, enabling this information to be mentally manipulated [12], is related to engagement in unhealthy lifestyle behaviours such as overeating [13] and smoking [14]. Furthermore, executive functioning is inversely related to cognitive stress reactivity [15] and perception [16]. Executive functioning encompasses inhibition, task switching and working memory [17]. These functions are localised in the prefrontal cortex, a key area modulating the stress-activated hypothalamic-pituitary-adrenal gland (HPA) axis [18]. Because patients with COPD exhibit smaller hippocampal [19] and prefrontal cortex volumes [20] than healthy controls [21], COPD may negatively impact cognitive stress susceptibility.

Only one earlier study has investigated cognitive training in patients with COPD, which did not improve cognitive functioning [22]. However, this study had a relatively low training load and it specifically targeted attention, learning and logical-deductive thinking. In contrast, the above-mentioned literature indicates that working memory training (WMT) could improve cognitive performance, adherence to healthy lifestyle behaviours and cognitive stress susceptibility.

It is still unclear whether WMT can improve cognitive performance in COPD. Its effects in healthy older adult populations are equivocal [23, 24], and improved cognitive functioning on a trained task often generalises only poorly into other domains [25].

Many patients with COPD lead a relatively unhealthy lifestyle, characterised by persistent smoking (in over one-third of patients) [26], physical inactivity (e.g., reported step counts of $<3000$ steps day $^{-1}$, whereas 5000 steps $\cdot$ day $^{-1}$ has been defined as the threshold of being considered sedentary) [27] and poor dietary quality (intake of macro- and micronutrients that is lower than the recommended daily intake and lower than in non-COPD controls) [28-30]. Dual-process theories of cognitive functioning [31] imply that WMT could improve lifestyle behaviours by strengthening top-down behavioural control [13, 32]. These theories state that two distinct systems handle cognitive operations: an automated, unconscious system, which is responsible for executing relatively easy, well-known and/or highly automated cognitive processes, and a controlled, conscious system for more deliberate, controlled and conscious execution of harder cognitive tasks [33]. The interaction between these two determines the extent to which one's responses are automatic or controlled [13]. WMT is supposed to strengthen the former system [34], thereby increasing conscious control over one's actions and enabling one to more carefully consider the degree to which actions, among others related to health behaviours (see, for example, DAssEn et al. [13] and HouBEN et al. [35]) are opportune [33, 36]. To our knowledge, only one study has investigated the effects of WMT on dietary intake in otherwise healthy overweight individuals [37]; its effects on physical activity levels have not yet been investigated.

WMT could beneficially modulate cognitive stress susceptibility and perception: it attenuated the salivary cortisol stress response in patients with major depressive disorder [38] and improved functioning of the brain areas responsible for modulating cognitive stress. WMT increased prefrontal activity and connectivity [39], and cognitive training had beneficial effects on hippocampal activation [40].

The primary aim of the Cogtrain trial was to investigate whether a 12-week WMT programme followed by a 12-week maintenance programme could establish and maintain cognitive improvement in patients with COPD. Its secondary aims were to investigate the effects of the intervention on physical capacity and activity, dietary quality, cognitive stress susceptibility and perception, and the recall of prespecified healthy lifestyle goals. In addition, depression and anxiety were investigated as exploratory outcomes and are reported in the supplementary material.

\section{Methods}

\section{Study design}

The double-blind randomised placebo-controlled Cogtrain trial consisted of a 12-week home-based intensive WMT (phase 1) followed by 12 weeks of active follow-up with weekly booster sessions (phase 2). The placebo group received sham training sessions in both phases (see figure 1). Measurements took place at Maastricht University Medical Centre 1 week before baseline (T0), at baseline (T1), after phase 1 (T2) and after phase 2 (T3; see table 1). All four of these visits lasted for 2.5-3 h. The study was registered at ClinicalTrials.gov (NCT03073954) and the medical ethics committee at Maastricht University Medical Centre granted ethical approval (NL59883.068.17/MEC 173010). 


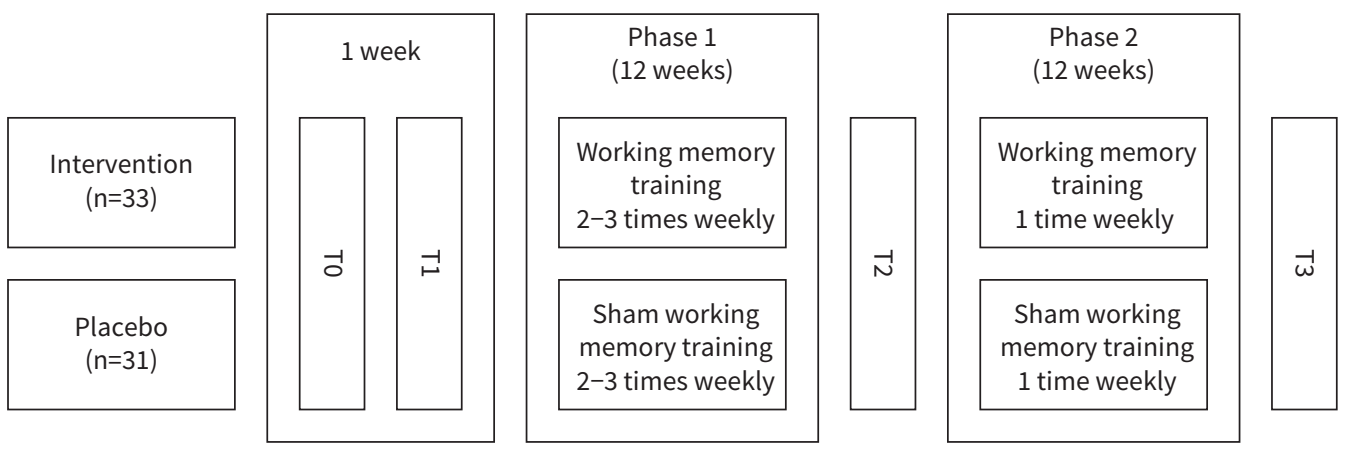

FIGURE 1 Study design.

Patients

Patients were eligible to participate in the study if they were aged 18 years or over and had a diagnosis of COPD based on the Global Initiative for Chronic Obstructive Lung Disease (GOLD) criteria [1]. Exclusion criteria were disease or disability limiting the ability to undergo neuropsychological testing and/or WMT (e.g., blindness, previous stroke or lack of hand control), neurological disorders (e.g., Alzheimer's or Parkinson's disease), insufficient mastery of the Dutch language, participation in an inpatient PR programme, not having access to a suitable device to complete the WMT on (i.e., laptop or personal computer), or participation in another interventional study over the course of the study period. Baseline

TABLE 1 Outcome measures and time points at which they were taken

\begin{tabular}{|c|c|c|c|c|}
\hline Instrument & TO & T1 & T2 & T3 \\
\hline \multicolumn{5}{|l|}{ Baseline demographic and clinical characteristics } \\
\hline Age, sex, educational level, smoking status & $\mathrm{x}$ & & & \\
\hline Spirometry & $\mathrm{x}$ & & & \\
\hline Manipulation check & & $\mathrm{x}$ & $\mathrm{x}$ & \\
\hline Working memory span & & $\mathrm{X}$ & $\mathrm{x}$ & \\
\hline Number of completed sessions & & $\mathrm{x}$ & $\mathrm{x}$ & \\
\hline \multicolumn{5}{|l|}{ Primary outcome measures } \\
\hline Cambridge Neuropsychological Test Automated Battery" & $\mathrm{x}$ & $\mathrm{x}$ & $\mathrm{x}$ & $\mathrm{x}$ \\
\hline Addenbrooke’s Cognitive Examination-Revised & & $\mathrm{x}$ & & \\
\hline \multicolumn{5}{|l|}{ Secondary outcome measures } \\
\hline \multicolumn{5}{|l|}{ Physical capacity and activity } \\
\hline 6-min walk test & & $\mathrm{x}$ & $\mathrm{x}$ & $\mathrm{x}$ \\
\hline Short Physical Performance Battery & & $\mathrm{x}$ & $\mathrm{x}$ & $\mathrm{x}$ \\
\hline Accelerometry & & $\mathrm{x}$ & $\mathrm{x}$ & $\mathrm{x}$ \\
\hline \multicolumn{5}{|l|}{ Dietary intake } \\
\hline Alternative Healthy Eating Index-2010 & $\mathrm{x}$ & & $\mathrm{x}$ & $\mathrm{x}$ \\
\hline \multicolumn{5}{|l|}{ Cognitive stress susceptibility and perception } \\
\hline Cortisol Awakening Response & & $\mathrm{x}$ & $\mathrm{x}$ & \\
\hline Perceived Stress Scale & & $\mathrm{x}$ & $\mathrm{x}$ & $\mathrm{x}$ \\
\hline Socially Evaluated Cold Pressor Test & & $\mathrm{x}$ & $\mathrm{x}$ & $\mathrm{x}$ \\
\hline Healthy lifestyle goal recall ${ }^{\natural}$ & & & $\mathrm{x}$ & $\mathrm{x}$ \\
\hline \multicolumn{5}{|l|}{ Exploratory outcome measures } \\
\hline \multicolumn{5}{|l|}{ Healthy lifestyle motivation } \\
\hline Behavioural Regulation of Exercise Questionnaire-2 & $\mathrm{x}$ & & & $\mathrm{x}$ \\
\hline Regulation of Eating Behaviours Scale & $\mathrm{x}$ & & & $x$ \\
\hline \multicolumn{5}{|l|}{ Psychological wellbeing } \\
\hline Beck Depression Inventory-II & & $\mathrm{x}$ & $\mathrm{x}$ & $\mathrm{x}$ \\
\hline Generalised Anxiety Disorder-7 & & $\mathrm{x}$ & $\mathrm{x}$ & $\mathrm{x}$ \\
\hline
\end{tabular}

T0: baseline minus 1 week; T1: baseline; T2: after the first phase (baseline+12 weeks); T3: after the second phase (baseline+24 weeks). \#: administration at T0 took place to compensate for learning effects. ": healthy lifestyle goals were set at $\mathrm{T} 1$. 
cognitive performance was not an inclusion criterium. Participants received EUR 50 upon completion of the entire study.

\section{Intervention}

During phase 1, participants received 30 emails with a link to a 20- to 25-min internet-based WMT session (i.e., two to three sessions weekly). Each session had to be completed on a patient's personal computer or laptop within $48 \mathrm{~h}$ of receiving the respective email or it would be marked as missed. Throughout the first phase, participants were invited to complete each session 2 days after completing the previous one. Participants were withdrawn from the study after missing six sessions. During phase 2 participants received 12 sessions (one per week) without a minimum number of sessions to be completed.

The WMT protocol has been used before [35, 41] and has been proven feasible and acceptable. It consisted of a visuospatial task, a backward digit span task and a letter span task, always presented in this order. See the supplementary material for a more detailed description of these tasks.

Participants in the intervention and placebo groups received the same tasks and the same number of trials. However, task difficulty was artificially held constant at three units throughout the intervention in the placebo group, whereas it was automatically adjusted on a trial-by-trial basis (adaptive WMT [37, 41]) in the intervention group. The treatments in the intervention and placebo groups were therefore different in that learning effects were only expected in the intervention group.

\section{Goal setting}

During T1, participants were informed about their physical activity and dietary quality (based on the relevant results obtained during T0), and they discussed dietary and physical activity goals with the researcher administering the test day. Physical activity goals were expressed as changes in number of steps per day, or a consolidation of the current number if already adequate at baseline. See the supplementary material for a more detailed description of the goal-setting procedure.

\section{Baseline demographic and clinical characteristics}

Sex, age, educational level, smoking status and lung function were collected and assessed at baseline. Lung function was assessed using the SpiroPerfect system (Welch Allyn, Skaneateles Falls, NY, USA) before and $15 \mathrm{~min}$ after administering $400 \mu \mathrm{g}$ salbutamol (Airomir Autohaler; Teva, Haarlem, the Netherlands). Additionally, Addenbrooke's Cognitive Examination-Revised (ACE-R) [42] was administered. This is a rapid (5-10 min) cognitive screening tool which incorporates five sub-domain scores (orientation/attention, memory, verbal fluency, language and visuospatial abilities) and is scored on a scale of $0-100$.

\section{Outcomes}

Manipulation check

Participants' maximum working memory span for each home-based WMT session was averaged over the three tests. Increased working memory span in the intervention group across phase 1 followed by a consolidation across phase 2 served as a validation of the intended effects of the intervention. Intervention compliance was assessed by the number of completed sessions.

\section{Primary outcomes}

The Cambridge Neuropsychological Test Automated Battery (CANTAB) [43] is a validated computerised cognitive function assessment tool. The Motor Screening Task (MOT; measuring psychomotor speed), Paired Associates Learning Task (PAL; gauging visuospatial associative learning), Stop-Signal Task (SST; inhibition), Reaction Time Task (RTI; psychomotor speed, attention), Delayed Match-to-Sample (DMS; recognition memory) and Spatial Working Memory Task (SWM; working memory, executive functioning) were administered in order to investigate a wide range of cognitive parameters (see supplementary Table S1). The battery took 45-60 min to complete. Administration at T0 served to account for potential learning effects; data of T1 through T3 were used in the analyses.

\section{Secondary outcomes}

Healthy lifestyle goal recall

At T2 and T3 participants were asked to recall the assessment procedure for their physical activity and dietary intake during earlier visits ("procedure") and the specific healthy lifestyle goals that had been set at T1 (“content”). See the supplementary material for a more detailed description of the healthy lifestyle goal recall procedure. 


\section{Physical capacity and activity}

Physical capacity was assessed using participants' obtained distance during the 6-min walk test [44] and their score on the Short Physical Performance Battery (SPPB) [45].

Physical activity was assessed by 7-day accelerometry (activPAL; PAL Technology, Glasgow, UK). The accelerometer was affixed to patients' upper legs using special water-resistant 3M Tegaderm tape. As such, the accelerometer was worn $24 \mathrm{~h}$ per day for 7 straight days, always consisting of 5 weekdays and 2 weekend days. No wear days were considered invalid because of insufficient wear time. Accelerometry data were subsequently processed using the activPAL software suite and quantified as number of steps per day and the amount of time spent sedentarily, standing up, walking and in high-intensity physical activity. Additionally, the number of sedentary breaks and sedentary bouts ( $\geqslant 30 \mathrm{~min}$ ) were assessed. The time participants spent in high-intensity physical activity, defined as $>110 \mathrm{steps} \cdot \mathrm{min}^{-1}$ [46, 47], was also calculated.

\section{Dietary quality}

Dietary intake was assessed using a 24-h recall paradigm. The results were entered into the "Eetmeter" tool of the Dutch Voedingscentrum. If participants knew the weight of their intake, this information was entered into the application, but portion sizes could also be entered in terms of numbers of tablespoons, ladles, cups, glasses, etc., based on an assumed size of a standard serving (e.g., a certain weight was assumed for a ladle of mashed potatoes, which could also be fine-tuned into a small, standard or large ladle). The "Eetmeter" application then calculated intake of a wide range of macro- and micronutrients. Dietary quality was quantified as the Alternative Healthy Eating Index (AHEI)-2010 score [48], based on intake of fruit, vegetables, wholewheat products, legumes/nuts, eicosapentaenoic and docosahexaenoic acid, the proportion of energy intake derived from polyunsaturated fatty acids, sodium, sweet drinks and juices, red and processed meat, and alcohol.

\section{Cognitive stress susceptibility and perception}

The socially evaluated cold pressor test (SECPT) [49] was administered as a measure of acute stress susceptibility; the cortisol awakening response (CAR) [50] served as a measure of chronic stress susceptibility. See the supplementary material for a more detailed description of the administration of these tests.

\section{Exploratory outcomes}

Healthy lifestyle motivation and psychological wellbeing served as exploratory outcomes. See the supplementary material for a more detailed description of these outcomes.

\section{Sample size and power}

The sample size calculation was performed using $G^{*}$ Power version 3.1.9.4 [51]. In a recent Canadian study examining the effects of cognitive training on cognitive decline, the authors reported an effect size of $f=0.475$ [52]. Combined with a two-tailed paired-samples t-test, an $\alpha$ of 0.05 and a power of $95 \%, 60$ individuals were required, or 30 per group. We anticipated 60 participants to complete, rather than to start, the trial so as to maintain adequate power throughout the study.

\section{Statistical analyses}

Data analysis was conducted according to the intention-to-treat principle using Stata 14 (StataCorp LP, College Station, TX, USA). Data of participants that prematurely quit the study were used up to the point of their withdrawal. Missing data were considered as missing at random and were not imputed. Two-sided p-values smaller than 0.05 were considered statistically significant. Analyses were conducted semi-blinded (i.e., participant allocation was revealed as "group 0" or "group 1", but not which of those was the intervention group), except for the home-based WMT analyses, given the nature of the data. The WMT analyses were conducted after all other analyses had been run.

The normality of all outcome variables was checked. Descriptive statistics of normally distributed variables were expressed as mean $\pm_{\mathrm{SD}}$; those of non-normally distributed variables as median (inter-quartile range).

Baseline between-group differences in working memory capacity were tested using independent-samples t-tests. Working memory capacity development in the intervention group across phase 1 (i.e., session 1 versus session 30) and phase 2 (i.e., session 31 versus session 42) were tested using one-sample t-tests with the values at sessions 1 and 31 as test value and the values at sessions 30 and 42 as dependent variables, respectively. 
Two-way repeated measures analyses of variance were conducted to compare differences over time and between the groups. Time point (T1-T3) and group (intervention versus placebo) were entered as independent variables. Phase 1 (i.e., T1 versus T2) and phase 2 (i.e., T2 versus T3) were analysed separately. Time, group and the timexgroup interaction were entered as independent variables. Age, sex and educational level were entered as covariates into the analyses with the cognitive parameters as dependent variables. Because these analyses are robust against a violation of the assumption of normality when the sample size exceeds 50 [53], non-normally distributed data were not transformed.

Results

Out of 301 patients assessed for eligibility, 68 were enrolled in the trial of whom 64 were randomised to the intervention $(n=33)$ or placebo group $(n=31)$ (see figure 2).

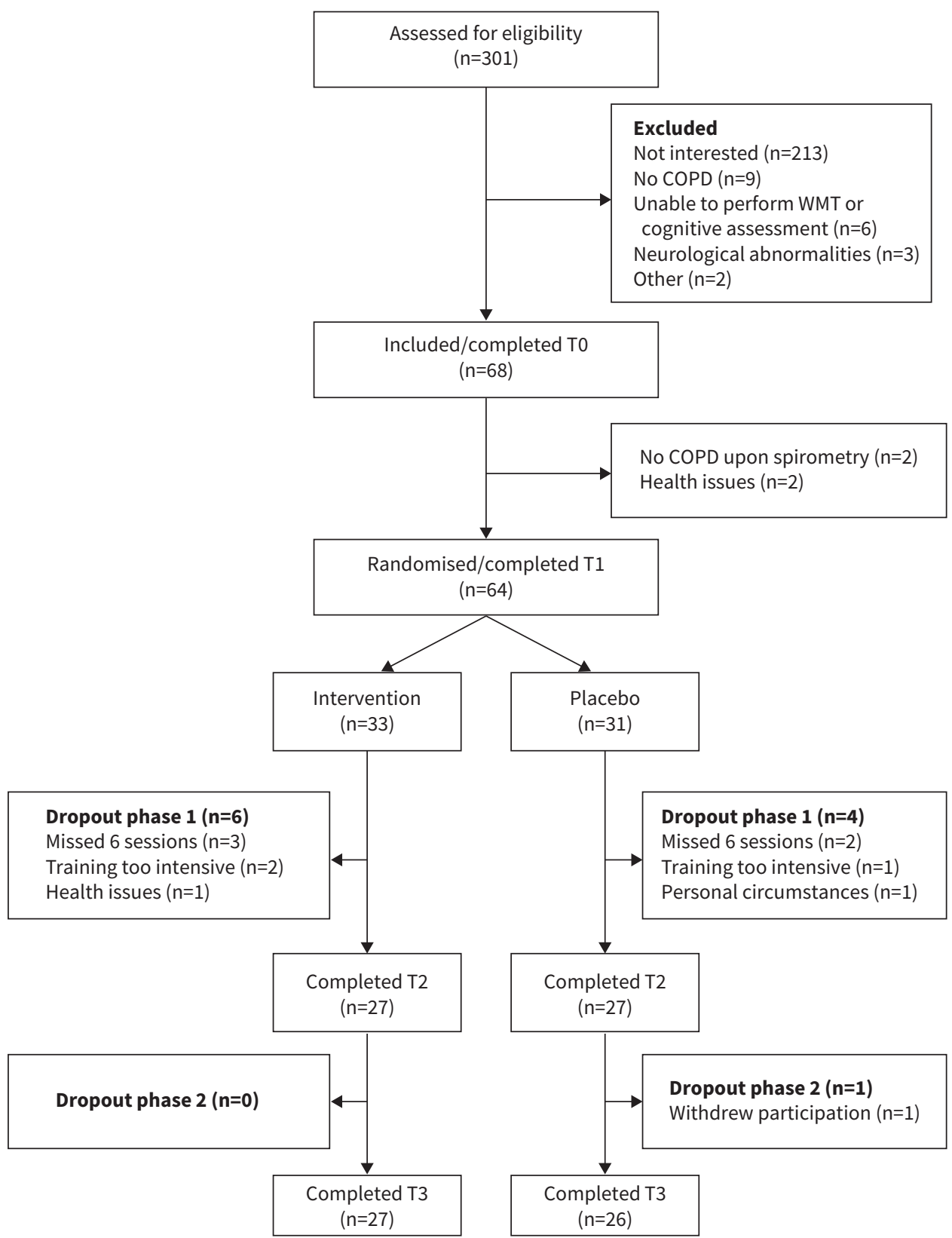

FIGURE 2 Study flowchart. WMT: working memory training. 


\begin{tabular}{|c|c|c|c|c|}
\hline & \multicolumn{2}{|c|}{ Intervention } & \multicolumn{2}{|c|}{ Placebo } \\
\hline & $\mathrm{n}$ & & $\mathrm{n}$ & \\
\hline Age (years) & 33 & $66.0 \pm 6.8$ & 31 & $66.4 \pm 7.8$ \\
\hline Sex (male/female) & 33 & $13 / 20$ & 31 & $16 / 15$ \\
\hline Educational level & 33 & & 31 & \\
\hline Primary school & & $2(6.1 \%)$ & & $0(0.0 \%)$ \\
\hline Initial vocational education & & $0(0.0 \%)$ & & $3(9.8 \%)$ \\
\hline High school & & $10(30.3 \%)$ & & $10(32.3 \%)$ \\
\hline Intermediate vocational education & & $6(18.2 \%)$ & & $8(25.8 \%)$ \\
\hline Higher vocational education & & $12(36.4 \%)$ & & $10(32.3 \%)$ \\
\hline Academic & & $3(9.1 \%)$ & & $0(0.0 \%)$ \\
\hline Smoking status (never/current/former) & 33 & $8 / 3 / 22$ & 31 & $6 / 6 / 19$ \\
\hline \multicolumn{5}{|l|}{ Lung function } \\
\hline GOLD stage (I/II/III/IV) & 31 & $8 / 11 / 11 / 1$ & 30 & $5 / 15 / 7 / 3$ \\
\hline $\mathrm{FEV}_{1}(\mathrm{~L})$ & 31 & $1.75 \pm 0.76$ & 30 & $1.69 \pm 0.69$ \\
\hline $\mathrm{FEV}_{1}$ (\% predicted) & 31 & $58.5(44.7-82.5)$ & 30 & $60.6(44.9-71.8)$ \\
\hline FVC (L) & 31 & $3.55 \pm 1.03$ & 30 & $3.49 \pm 0.98$ \\
\hline $\mathrm{FEV}_{1} / \mathrm{FVC}(\%)$ & 31 & $49.0 \pm 14.7$ & 30 & $48.4 \pm 14.7$ \\
\hline ACE-R (0-100) & 33 & $88(82-93)$ & 31 & $88(83-94)$ \\
\hline
\end{tabular}

\section{Baseline demographic and clinical characteristics}

At baseline, the study population (45\% male, aged $66.2 \pm 7.2$ years) was characterised by on average moderate airflow limitation (median forced expiratory volume in $1 \mathrm{~s} 60.6 \%$ predicted, inter-quartile range 45.6-77.0) and impaired exercise capacity (6-min walk distance $457.7 \pm 84.7 \mathrm{~m}$ ) but a relatively high physical activity level for this population of chronically diseased patients $\left(7525 \pm 3254\right.$ steps $^{- \text {day }^{-1}}$ ). Baseline cognitive functioning was relatively poor (median ACE-R score 88, inter-quartile range 83-94) compared to earlier reported normal values [42] (see table 2). No parameters were different between the groups at baseline, except the Beck Depression Inventory-II (BDI-II) $(\mathrm{p}=0.002)$ and the Regulation of Eating Behaviour (REBS) amotivation scale ( $\mathrm{p}=0.027)$.

\section{Manipulation check}

During phase 1, six participants (18.2\%) dropped out of the intervention group and four (12.9\%) dropped out of the placebo group (see figure 2). Participants in the intervention group completed on average 23.6 \pm 6.2 out of 30 WMT sessions during phase 1 and $7.7 \pm 3.6$ out of 12 during phase 2 , compared to $27.4 \pm 9.7$ and $5.0 \pm 2.3$, respectively, in the placebo group. The mean working memory span was not significantly different between the groups at baseline $(p=0.888)$. The working memory span of the intervention group increased significantly during phase $1(\mathrm{p}<0.001)$ and remained stable during phase $2(\mathrm{p}=0.399)$ (see figure 3).

\section{Primary outcomes}

Table 3 reports the descriptive statistics of the primary outcome measures; table 4 reports their effect sizes.

Across phase 1 and phase 2, the intervention had no effect on five of the six CANTAB tests. The only significant beneficial effect was found on the "probability of an error given error" parameter of the DMS, which tended to decrease in the intervention group $(p=0.077)$ and remained stable in the placebo group $(p=0.223)$, leading to a significant interaction effect $(p=0.038)$. Across phase 2 , there was only a significant positive effect on the five-choice movement time of the RTI, which decreased in the intervention group $(p=0.016)$ and remained stable in the placebo group $(p=0.303)$, also leading to a significant interaction effect $(\mathrm{p}=0.017)$.

\section{Secondary outcomes}

Table 5 reports the descriptive statistics of the secondary outcome measures; table 6 reports their effect sizes. 


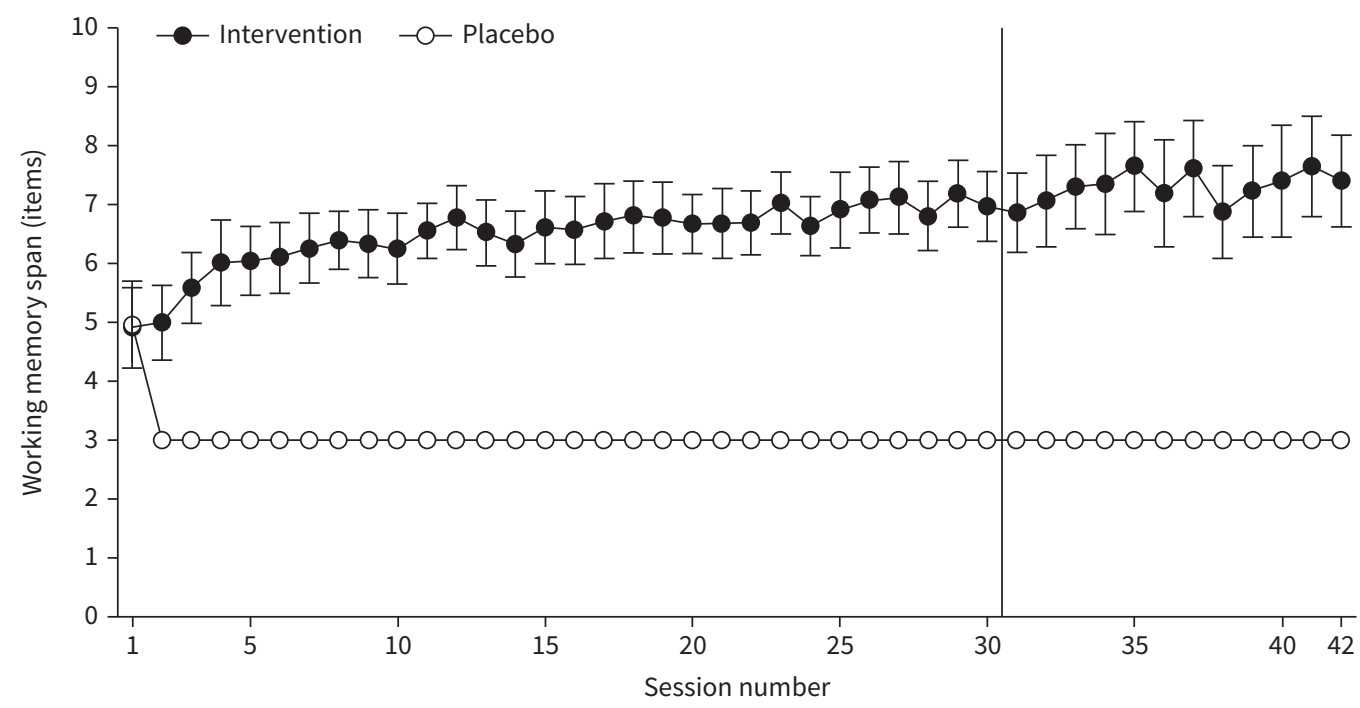

FIGURE 3 Working memory span over the course of the first (session 1-30) and second phase (session 31-42) of the working memory training.

Healthy lifestyle goal recall

The intervention had no significant effects on the recollection of the goal-setting procedure or the contents of the set goals at any time point.

TABLE 3 Descriptive statistics of the primary (Cambridge Neuropsychological Test Automated Battery, CANTAB) outcome measures

\begin{tabular}{|c|c|c|c|c|c|c|c|}
\hline \multirow[t]{2}{*}{ Test } & \multirow[t]{2}{*}{ Measure } & \multicolumn{2}{|c|}{$\mathrm{T} 1$} & \multicolumn{2}{|c|}{$\mathrm{T} 2$} & \multicolumn{2}{|c|}{ T3 } \\
\hline & & Intervention & Placebo & Intervention & Placebo & Intervention & Placebo \\
\hline MOT & Mean latency (ms) & $896(696-1031)$ & $894(761-1056)$ & $903(755-980)$ & 865 (736-961) & $860(771-940)$ & 827 (745-976) \\
\hline \multirow[t]{3}{*}{ PAL } & Total errors $(n)$ & $13.0(11.0-20.0)$ & $13.0(8.0-17.0)$ & $13.0(10.0-16.0)$ & $13.0(11.0-17.0)$ & $13.0(9.0-18.0)$ & $13.0(9.0-21.0)$ \\
\hline & Adjusted total errors (n) & $20.0(12.0-39.0)$ & $19.0(10.0-41.0)$ & $14.0(10.0-42.0)$ & $16.0(11.0-24.0)$ & $17.0(9.0-36.0)$ & $14.5(9.0-21.0)$ \\
\hline & First attempt memory score & $9.76 \pm 3.83$ & $10.55 \pm 4.56$ & $10.89 \pm 4.34$ & $10.56 \pm 3.53$ & $10.88 \pm 3.17$ & $12.27 \pm 3.06$ \\
\hline SST & $\begin{array}{l}\text { Stop-signal reaction time } \\
(\mathrm{ms})\end{array}$ & $244(220-272)$ & $240(213-275)$ & $258(240-276)$ & $236(215-262)$ & $249(224-267)$ & $223(208-253)$ \\
\hline \multirow[t]{4}{*}{ RTI } & $\begin{array}{l}\text { Median simple reaction time } \\
\text { (ms) }\end{array}$ & $328(308-349)$ & $340(316-366)$ & $331(311-350)$ & $349(325-365)$ & $341(320-361)$ & $361(338-386)$ \\
\hline & $\begin{array}{l}\text { Mean simple movement } \\
\text { time (ms) }\end{array}$ & $253(216-286)$ & $243(207-302)$ & $250(225-288)$ & $264(229-313)$ & $272(214-303)$ & $272(223-326)$ \\
\hline & $\begin{array}{l}\text { Median five-choice reaction } \\
\text { time (ms) }\end{array}$ & $399(366-429)$ & $392(370-412)$ & $399(366-426)$ & $392(358-425)$ & $425(382-441)$ & $419(379-450)$ \\
\hline & $\begin{array}{l}\text { Mean five-choice movement } \\
\text { time (ms) }\end{array}$ & $290(240-313)$ & $283(245-331)$ & $282(271-339)$ & $309(258-331)$ & $272(239-312)$ & $307(250-336)$ \\
\hline \multirow[t]{3}{*}{ DMS } & Correct responses (\%) & $85.0(80.0-90.0)$ & $85.0(75.0-90.0)$ & $90.0(85.0-95.0)$ & $85.0(75.0-95.0)$ & $85.0(80.0-95.0)$ & $85.0(77.5-92.5)$ \\
\hline & Median correct latency (ms) & $3027(2494-3370)$ & $3294(2593-3488)$ & $3003(2478-4305)$ & 3255 (2475-4342) & $3295(2411-4243)$ & $3159(2534-4490)$ \\
\hline & $\begin{array}{l}\text { Probability of error given } \\
\text { error (\%) }\end{array}$ & $0.0(0.0-20.0)$ & $0.0(0.0-16.7)$ & $0.0(0.0-0.0)$ & $0.0(0.0-20.0)$ & $0.0(0.0-25.0)$ & $0.0(0.0-21.0)$ \\
\hline \multirow[t]{5}{*}{ SWM } & Between-errors ( $\mathrm{n})$ & $16.0(6.0-21.0)$ & $15.0(6.0-23.0)$ & $15.0(7.0-20.0)$ & $17.0(10.0-20.0)$ & $16.0(9.0-21.0)$ & $12.0(4.0-19.0)$ \\
\hline & Between-errors 4 boxes ( $n$ ) & $0.0(0.0-1.0)$ & $0.0(0.0-2.0)$ & $0.0(0.0-0.0)$ & $0.0(0.0-1.0)$ & $0.0(0.0-2.0)$ & $0.0(0.0-0.0)$ \\
\hline & Between-errors 6 boxes ( $n$ ) & $3.0(0.0-6.0)$ & $4.0(0.0-7.0)$ & $4.0(1.0-7.0)$ & $4.0(1.0-7.0)$ & $2.0(0.0-7.0)$ & $2.0(0.0-6.0)$ \\
\hline & Between-errors 8 boxes ( $n$ ) & $11.0(4.0-15.0)$ & $10.0(5.0-14.0)$ & $10.0(5.0-14.0)$ & $11.0(6.0-14.0)$ & $12.0(8.0-14.0)$ & $10.0(3.0-14.0)$ \\
\hline & Strategy & $8.0(7.0-10.0)$ & $8.0(6.0-10.0)$ & $9.0(7.0-10.0)$ & $9.0(6.0-10.0)$ & $8.0(6.0-9.0)$ & $9.0(7.0-9.0)$ \\
\hline
\end{tabular}

Data presented as mean \pm SD or median (inter-quartile range) depending on the normality of the distribution. MOT: motor orientation task; PAL: paired associates learning; SST: stop-signal task; RTI: reaction time task; DMS: delayed match-to-sample; SWM: spatial working memory. 


TABLE 4 Effect sizes of the primary (Cambridge Neuropsychological Test Automated Battery, CANTAB) outcome
measures

TABLE 5 Descriptive statistics of the secondary outcomes of the intervention

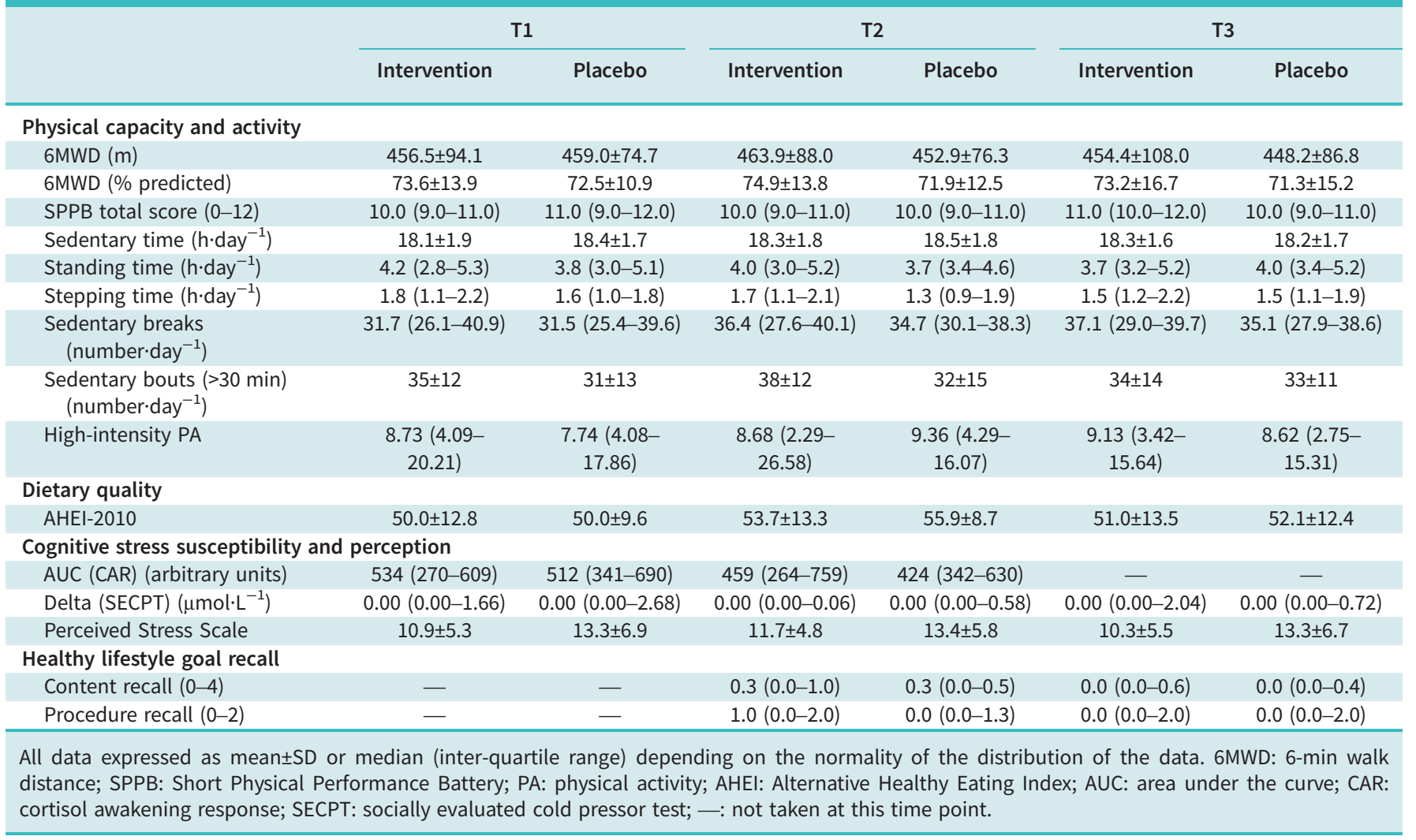




\begin{tabular}{|c|c|c|c|c|c|c|}
\hline \multirow[t]{2}{*}{ Measure } & \multicolumn{3}{|c|}{ Phase 1} & \multicolumn{3}{|c|}{ Phase 2} \\
\hline & $F$ time & $F$ group & $F$ int. & $F$ time & $F$ group & $F$ int. \\
\hline \multicolumn{7}{|l|}{ Physical capacity and activity } \\
\hline 6MWD (m) & 0.10 & 0.05 & 0.61 & 1.00 & 0.11 & 0.20 \\
\hline 6MWD (\% predicted) & 0.25 & 0.46 & 0.70 & 1.23 & 0.39 & 0.29 \\
\hline SPPB total score (0-12) & 0.05 & 0.03 & 0.00 & 0.30 & 0.96 & 1.48 \\
\hline Sedentary time $\left(\mathrm{h} \cdot\right.$ day $\left.^{-1}\right)$ & 0.00 & 1.16 & 1.17 & 1.24 & 2.74 & 0.80 \\
\hline Standing time $\left(\mathrm{h} \cdot \mathrm{day}^{-1}\right)$ & 0.60 & 0.10 & 0.07 & 0.07 & 0.07 & 0.10 \\
\hline Stepping time $\left(\mathrm{h} \cdot \mathrm{day}^{-1}\right)$ & 0.35 & 1.04 & 0.03 & 0.19 & 1.14 & 2.01 \\
\hline Sedentary breaks (number $\cdot$ day $^{-1}$ ) & $9.14^{\star \star}$ & 0.43 & 0.50 & 0.05 & 2.17 & 0.18 \\
\hline Sedentary bouts (>30 min) (number $\cdot$ day $^{-1}$ ) & 0.42 & 1.29 & 0.03 & 0.99 & 2.21 & 0.99 \\
\hline High-intensity PA & 2.45 & 0.20 & 0.12 & 0.26 & 0.64 & 0.11 \\
\hline \multicolumn{7}{|l|}{ Dietary quality } \\
\hline AHEI-2010 & $5.04^{\star}$ & 0.11 & 0.26 & 1.87 & 0.31 & 0.00 \\
\hline \multicolumn{7}{|l|}{ Cognitive stress susceptibility and perception } \\
\hline AUC (CAR) (arbitrary units) & 0.02 & 0.14 & 0.74 & - & - & - \\
\hline Delta $(\mathrm{SECPT})\left(\mu \mathrm{mol} \cdot \mathrm{L}^{-1}\right)$ & $6.05^{\star}$ & 0.08 & 0.14 & 0.93 & 1.46 & $4.19^{*}$ \\
\hline Perceived Stress Scale & 0.52 & 2.41 & 0.10 & 2.55 & 2.27 & 1.05 \\
\hline \multicolumn{7}{|l|}{ Healthy lifestyle goal recall } \\
\hline Procedure recall (0-4) & - & - & - & 0.00 & 1.63 & 1.02 \\
\hline Content recall (0-2) & - & - & - & 3.16 & 2.14 & 0.15 \\
\hline
\end{tabular}

\section{Physical capacity and activity}

The intervention did not influence participants' physical capacity and activity. The number of sedentary breaks per day increased significantly across both groups across phase $1(p=0.004)$. However, this does not indicate an effect of the intervention, and the total amount of time spent sedentarily or the number of prolonged sedentary bouts were not significantly affected.

\section{Dietary quality}

The intervention did not affect participants' dietary quality, but the AHEI-2010 score improved significantly across phase 1 in both groups $(\mathrm{p}=0.029)$.

\section{Cognitive stress susceptibility and perception}

Across phase 1 , the intervention did not significantly affect any stress susceptibility parameter. The SECPT-induced change in cortisol level decreased in both groups $(\mathrm{p}=0.019)$, indicating overall decreased stress reactivity. The magnitude of the SECPT-induced change in cortisol levels increased significantly in the intervention group across phase 2 ( $\mathrm{p}=0.033$ ), indicating increased stress reactivity, whereas it remained stable in the placebo group ( $\mathrm{p}=0.462)$, leading to a significant interaction effect $(\mathrm{p}=0.047)$. This indicates an effect of the intervention.

\section{Exploratory outcomes}

See the supplementary material for the results of the motivational and psychological exploratory outcome measures.

\section{Discussion}

This clinical trial aimed to investigate whether WMT could improve cognitive performance, adherence to healthy lifestyle behaviours, recall of prespecified healthy lifestyle goals and cognitive stress susceptibility and perception in patients with COPD. The WMT was feasible as working memory capacity on the trained tasks improved significantly in the intervention group across phase 1 and remained stable in phase 2 . Nevertheless, this did not improve CANTAB task performance. The intervention significantly affected only 1 of 17 CANTAB parameters in both phases of the intervention, and these parameters were different in both phases. Overall, the intervention thus did not improve any of the six investigated cognitive domains. This is in line with earlier studies: cognitive training can improve performance on tests that are identical or similar to those that were trained [23,54], but these improvements generalise poorly into other cognitive tests or 
domains, and they are poorly maintained in the longer term [25]. The only earlier trial investigating cognitive training in patients with COPD did not significantly improve cognitive performance either [22].

Baseline cognitive performance was highly variable: the median ACE-R score of 88 indicates relatively poor performance, but the highest-performing quartile of participants, scoring 94 and above, showed normal cognitive performance. This, along with the fact that it is unknown whether WMT is more beneficial for those with poor baseline cognitive performance (because of their larger room for improvement) or for highly performing patients (because of their arguably higher cognitive and neural plasticity and therefore higher learning potential) may have contributed to the overall lack of effects of WMTs.

The effects of the intervention on healthy lifestyle outcomes, psychological wellbeing and healthy lifestyle motivation were also limited. This is not surprising, as cognitive improvement by WMT, which was hypothesised to enable a healthier lifestyle, was not attained in the first place. Regarding physical activity, the intervention improved only one of three indices of a sedentary lifestyle. Intrinsic motivation towards healthy eating improved, but not actual dietary quality (which interestingly improved in both groups across phase 1). WMT has improved healthy lifestyle behaviours such as caloric intake [13, 55] or alcohol intake [35] in earlier trials, but these selectively included certain subgroups that stood to gain a lot from the interventions, such as overweight participants or substance abusers. These patients were therefore much more likely to improve than our patients with relatively high levels of physical activity and a relatively healthy diet.

Previous interventions aiming to improve dietary quality have combined WMT with education (e.g., DASSEN et al. [13]), to make sure that WMT-induced increased abilities to override automatic responses would be aimed at the appropriate responses. This is relevant as $28.5 \%$ [56] and $30.2 \%$ [57] of patients with COPD have poor health literacy. In other words, patients might simply "not know that they don't know" what is and is not healthy. Additionally, a motivational component such as motivational interviewing could have contributed to participants' willingness to change, thereby also increasing adherence to healthy lifestyle behaviours in the longer term $[58,59]$. The lack of educational or motivational components in the current trial might have contributed to its inability to significantly improve dietary quality.

Weaknesses also include the relatively low response rate (for instance because the high study load deterred potential participants or because they did not own a computer) and the fact that the foreseen sample size was not attained (53 instead of 60 patients completed the trial). However, given the pattern of the results, seven additional patients would probably not have made a larger difference. Moreover, the sample size was comparable to other recently published studies [60,61]. The study may also have suffered from selection bias towards relatively highly motivated patients with a healthier lifestyle and remarkably high levels of physical activity for a population of patients with chronic disease. This may have contributed to the lack of effects on physical activity. Furthermore, although the detrimental impact of smoking on cognitive performance has been well documented $[62,63]$, this or the effects of WMT on smoking cessation, as part of healthy lifestyle improvement, have not been further investigated in the current study. The equal division of current, former and never-smokers between the intervention and placebo groups was, however, verified at baseline.

Strengths of the trial are its rigorous double-blind, placebo-controlled design and its comprehensive neuropsychological assessment, which gives a much more comprehensive indication of cognitive performance than frequently used screening tools such as the Mini-Mental State Examination or the Montreal Cognitive Assessment [8, 64]. The study had relatively few inclusion and exclusion criteria, aiming to include a representative population of community-dwelling COPD patients. Furthermore, the dropout rate was equivalent to or lower than comparable studies [65, 66].

In conclusion, WMT in its current form was not effective in improving cognitive performance, healthy lifestyle behaviours, cognitive stress susceptibility and perception, healthy lifestyle goal recall, healthy lifestyle motivation or psychological wellbeing in community-dwelling patients with COPD. Future research should consider incorporating additional interventional components, such as education, and investigate specific at-risk subgroups to examine the effectivity of WMT.

Author contributions: H.R. Gosker, K. Houben and A.M.W.J. Schols conceived and designed the study. M. van Beers, S.W. Mount and L. Schuurman collected the data. M. van Beers, H.R. Gosker and A.M.W.J. Schols analysed and interpreted the data. M. van Beers wrote the initial draft of the manuscript. All authors reviewed and edited the draft manuscript. All authors critically revised the article and gave final approval of this version to be published. 
All authors had full access to all data, including statistical reports and tables, in the study, and can take full responsibility for the integrity of the data and the accuracy of the data analysis.

Provenance: Submitted article, peer reviewed.

This study is registered at www.clinicaltrials.gov with identifier number NCT03073954. The authors will make anonymised patient data available upon reasonable request.

Conflict of interest: None declared.

Support statement: This work was supported by the Maastricht University Interfaculty Program "Eatwell" and through the JPI HDHL project "Ambrosiac" by ZonMW (project number 529051006). These funding bodies were not involved in the study design; in the collection, analysis or interpretation of data; in the writing of the report; or in the decision to submit the article for publication. The researchers were independent from the funders. Funding information for this article has been deposited with the Crossref Funder Registry.

References

1 Global Initiative for Chronic Obstructive Lung Disease (GOLD). Global Strategy for the Diagnosis, Management and Prevention of Chronic Obstructive Pulmonary Disease. 2020. Available from: http://goldcopd.org/

2 Van den Borst B, Slot IGM, Hellwig VACV, et al. Loss of quadriceps muscle oxidative phenotype and decreased endurance in patients with mild-to-moderate COPD. J Appl Physiol 2013; 114: 1319-1328.

3 Cebron Lipovec N, Beijers RJ, Van den Borst B, et al. The prevalence of metabolic syndrome in chronic obstructive pulmonary disease: a systematic review. COPD 2016; 13: 399-406.

4 Trajanoska K, Schoufour JD, Darweesh SKL, et al. Sarcopenia and its clinical correlates in the general population: the Rotterdam Study. J Bone Miner Res 2018; 33: 1209-1218.

5 Ouellette DR, Lavoie KL. Recognition, diagnosis and treatment of cognitive and psychiatric disorders in patients with COPD. Int J COPD 2017; 12: 639-650.

6 Yohannes AM, Chen W, Moga AM, et al. Cognitive impairment in chronic obstructive pulmonary disease and chronic heart failure: a systematic review and meta-analysis of observational studies. JAMDA 2017; 18: 451. e1-451.e11.

7 Roncero C, Campuzano Al, Quintano JA, et al. Cognitive status among patients with chronic obstructive pulmonary disease. Int J COPD 2016; 11: 543-551.

8 Cleutjens FAHM, Spruit MA, Ponds RWHM, et al. Cognitive impairment and clinical characteristics in patients with chronic obstructive pulmonary disease. Chron Respir Dis 2018; 15: 91-102.

9 Cleutjens FAHM, Franssen F, Spruit MA, et al. Domain-specific cognitive impairment in patients with COPD and control subjects. Int J Chron Obstruct Pulmon Dis 2017; 12: 1-11.

10 Van Beers M, Janssen DJA, Gosker HR, et al. Cognitive impairment in chronic obstructive pulmonary disease: disease burden, determinants and possible future interventions. Expert Rev Respir Med 2018; 12: 1061-1074.

11 Andrianopoulos V, Gloeckl R, Vogiatzis I, et al. Cognitive impairment in COPD: should cognitive evaluation be part of respiratory assessment? Breathe 2017; 13: e1-e9.

12 Baddeley A. Working memory. Science 1992; 255: 556-559.

13 Dassen FCM, Houben K, Van Breukelen GJP, et al. Gamified working memory training in overweight individuals reduces food intake but not body weight. Appetite 2018; 124: 89-98.

14 Day AM, Kahler CW, Metrik J, et al. Working memory moderates the association between smoking urge and smoking lapse behavior after alcohol administration in a laboratory analogue task. Nicotine Tob Res 2015; 17: 1173-1177.

15 Roiland RA, Lin F, Phelan C, et al. Stress regulation as a link between executive function and pre-frailty in older adults. J Nutr Health Aging 2015; 19: 828-838.

16 Shields GS, Moons WG, Slavich GM. Better executive function under stress mitigates the effects of recent life stress exposure in young adults. Stress 2017; 20: 75-85.

17 Diamond A. Executive functions. Annu Rev Psychol 2013; 64: 135-168.

18 McEwen BS, Bowles NP, Gray JD, et al. Mechanisms of stress in the brain. Nat Neurosci 2015; 18: 1353-1363.

19 Esser RW, Stoeckel MC, Kirsten A, et al. Structural brain changes in patients with COPD. Chest 2016; 149: 426-434.

20 Chen J, Lin I-T, Zhang $\mathrm{H}$, et al. Reduced cortical thickness, surface area in patients with chronic obstructive pulmonary disease: a surface-based morphometry and neuropsychological study. Brain Imaging Behav 2016; 10: 464-476.

21 Arnsten AFT. Stress weakens prefrontal networks: molecular insults to higher cognition. Nat Neurosci 2015; 18: $1376-1385$.

22 Antonelli Incalzi R, Corsonello A, Trojano L, et al. Cognitive training is ineffective in hypoxemic COPD: a six-month randomised controlled trial. Rejuvenation Res 2008; 11: 239-250. 
$23 \mathrm{Hu} \mathrm{M}$, Wu X, Shu X, et al. Effects of computerised cognitive training on cognitive impairment: a meta-analysis. J Neurol 2021; 268: 1680-1688.

24 Fan BJY, Wong RYM. Effect of cognitive training on daily function in older people without major neurocognitive disorder: a systematic review. Geriatrics 2019; 4: 44.

25 Melby-Lervag M, Redick TS, Hulme C. Working memory training does not improve performance on measures of intelligence or other measures of 'far transfer': evidence from a meta-analytic review. Perspect Psychol Sci 2016; 11: 512-534.

26 Agusti AG, Calverley PMA, Celli BR, et al. Characterisation of COPD heterogeneity in the ECLIPSE cohort. Respir Res 2010; 11: 122.

27 Kantorowski A, Wan ES, Homsy D, et al. Determinants and outcomes of change in physical activity in COPD. ERJ Open Res 2018; 4: 00054-2018.

28 Laudisio A, Costanzo L, Di Gioia C, et al. Dietary intake of elderly outpatients with chronic obstructive pulmonary disease. Arch Gerontol Geriatr 2016; 64: 75-81.

29 Chambaneau A, Filaire M, Jubert L, et al. Nutritional intake, physical activity and quality of life in COPD patients. Int J Sports Med 2016; 37: 730-737.

30 Van de Bool C, Mattijssen-Verdonschot C, Van Melick PPMJ, et al. Quality of dietary intake in relation to body composition in patients with chronic obstructive pulmonary disease eligible for pulmonary rehabilitation. Eur J Clin Nutr 2014; 68: 159-165.

31 Kahneman D. A perspective on judgment and choice: mapping bounded rationality. Am Psychol 2003; 58: 697-720.

32 Hofmann W, Friese M, Strack F. Impulse and self-control from a dual-systems perspective. Perspect Psychol Sci 2009; 4: 162-176.

33 Kahneman D. Thinking, Fast and Slow. New York, NY, Farrar, Straus and Giroux, 2011.

34 Evans JSBT, Stanovich KE. Dual-process theories of higher cognition: advancing the debate. Perspect Psychol Sci 2013; 8: 223-241.

35 Houben K, Wiers RW, Jansen A. Getting a grip on drinking behavior: training working memory to reduce alcohol abuse. Psychol Sci 2011; 22: 968-975.

36 Hofmann W, Schmeichel BJ, Baddeley A. Executive functions and self-regulation. Trends Cogn Sci 2012; 16: 174-180.

37 Houben K, Dassen FCM, Jansen A. Taking control: working memory training in overweight individuals increases self-regulation of food intake. Appetite 2016; 105: 567-574.

38 Jopling E, Gotlib IH, LeMoult J. Effects of working memory training on cognitive, affective and biological responses to stress in major depression: a novel cognitive bias modification protocol. J Affect Disord 2020; 265: 45-51.

39 Constantinidis C, Klingberg T. The neuroscience of working memory capacity and training. Nat Rev Neurosci 2016; 17: 438-449.

40 Rosen AC, Sugiura L, Kramer JH, et al. Cognitive training changes hippocampal function in mild cognitive impairment: a pilot study. J Alzheimer's Dis 2011; 26: 349-357.

41 Klingberg $\mathrm{T}$, Forssberg $\mathrm{H}$, Westerberg $\mathrm{H}$. Training of working memory in children with ADHD. J Clin Exp Neuropsychol 2002; 24: 781-791.

42 Mioshi E, Dawson K, Mitchell J, et al. The Addenbrooke's Cognitive Examination Revised (ACE-R): a brief cognitive test battery for dementia screening. Int J Geriatr Psychiatry 2006; 21: 1078-1085.

43 Cambridge Cognition. CANTAB (R). Cambridge Cognition, 2019. www.cantab.com

44 Troosters T, Gosselink R, Decramer M. Six minute walking distance in healthy elderly subjects. Eur Respir $J$ 1999; 14: 270-274.

45 Guralnik JM, Simonsick EM, Ferrucci L, et al. A short physical performance battery assessing lower extremity function: association with self-reported disability and prediction of mortality and nursing home admission. $J$ Gerontol Med Sci 1994; 49: M85-M94.

46 De Rooij BH, Van der Berg JD, Van der Kallen CJH, et al. Physical activity and sedentary behavior in metabolically healthy versus unhealthy obese and non-obese individuals - The Maastricht Study. PLoS ONE 2016; 11: e0154358.

47 Van der Berg JD, Stehouwer CDA, Bosma H, et al. Associations of total amount and patterns of sedentary behaviour with type 2 diabetes and the metabolic syndrome: the Maastricht Study. Diabetologia 2016; 59: 709-718.

48 Chiuve SE, Fung TT, Rimm EB, et al. Alternative dietary indices both strongly predict risk of chronic disease. $J$ Nutr 2012; 142: 1009-1018.

49 Schwabe L, Haddad L, Schachinger H. HPA axis activation by a socially evaluated cold-pressor test Psychoneuroendocrinology 2008; 33: 890-895.

50 Wüst $\mathrm{S}$, Wolf J, Hellhammer $\mathrm{DH}$, et al. The cortisol awakening response - normal values and confounds. Noise Health 2000; 7: 77-85. 

behavioral and biomedical sciences. Behav Res Methods 2007; 39: 175-191.

52 Gill DP, Gregory MA, Zou G, et al. The healthy mind, healthy mobility trial: a novel exercise program for older adults. Med Sci Sports Exerc 2016; 48: 297-306.

53 Pituch KA, Stevens JP. Applied Multivariate Statistics for the Social Sciences. 6th Edn. New York \& London, Routledge, 2016.

54 Mowszowski L, Lampit A, Walton CC, et al. Strategy-based cognitive training for improving executive functions in older adults: a systematic review. Neuropsychol Rev 2016; 26: 252-270.

55 Yang Y, Shields GS, Wu Q, et al. Cognitive training on eating behaviour and weight loss: a meta-analysis and systematic review. Obes Rev 2019; 20: 1628-1641.

56 O'Conor R, Muellers KA, Arvanitis M, et al. Effects of health literacy and cognitive abilities on COPD self-management behaviors: a prospective cohort study. Respir Med 2019; 160: 105630.

57 Muellers KA, Chen L, O'Conor R, et al. Health literacy and medication adherence in COPD patients: when caregiver presence is not sufficient. COPD 2019; 16: 362-367.

58 Teixeira PJ, Carraca EV, Markland D, et al. Exercise, physical activity and self-determination theory: a systematic review. Int J Behav Nutr Phys Act 2012; 9: 78.

59 Leblanc V, Bégin C, Hudon A-M, et al. Effects of a nutritional intervention program based on the self-determination theory and promoting the Mediterranean diet. Health Psychol Open 2016; 3: 2055102915622094

60 Lee GJ, Bang HJ, Lee KM, et al. A comparison of the effects between 2 computerised cognitive training programs, Bettercog and COMCOG, on elderly patients with $\mathrm{MCl}$ and mild dementia: a single-blind randomised controlled study. Medicine 2018; 97: e13007.

61 Park J, Kim S-E, Kim E-J, et al. Effect of 12-week home-based cognitive training on cognitive function and brain metabolism in patients with amnestic mild cognitive impairment. Clin Interv Aging 2019; 14: 1167-1175.

62 Swan GE, Lessov-Schlaggar CN. The effects of tobacco smoke and nicotine on cognition and the brain. Neuropsychol Rev 2007; 17: 259-273.

63 Campos MW, Serebrisky D, Castaldelli-Maia JM. Smoking and cognition. Curr Drug Abuse Rev 2016; 9: 1-4.

64 Pierobon A, Bottelli ES, Ranzini L, et al. COPD patients' self-reported adherence, psychosocial factors and mild cognitive impairment in pulmonary rehabilitation. Int J COPD 2017; 12: 2059-2067.

65 Han JW, Son KL, Byun HJ, et al. Efficacy of the Ubiquitous Spaced Retrieval-based Memory Advancement and Rehabilitation Training (USMART) program among patients with mild cognitive impairment: a randomised controlled crossover trial. Alzheimer's Res Ther 2017; 9: 39.

66 Souders DJ, Boot WR, Blocker K, et al. Evidence for narrow transfer after short-term cognitive training in older adults. Front Aging Neurosci 2017; 9: 41. 